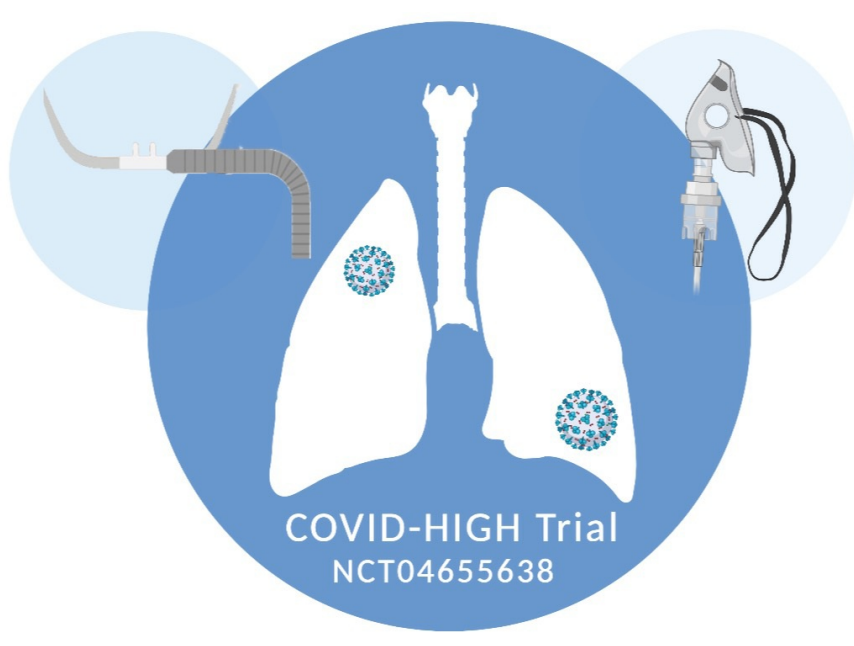

\title{
The COVID-HIGH RANDOMIZED CLINICAL TRIAL
}

Andrea Cortegiani ${ }^{1}$, Mariachiara Ippolito, CLAUDIA CRIMI

1 University of Palermo

Funding: The author(s) received no specific funding for this work.

Potential competing interests: The author(s) declared that no potential competing interests exist.

\section{Abstract}

The aim of this unblinded parallel-group randomized multicenter clinical trial is to compare the clinical effectiveness of high flow nasal therapy (HFNT) with conventional oxygen therapy (COT) in patients with confirmed COVID-19 related acute hypoxemic respiratory failure.

ClinicalTrials.gov Identifier: NCT04655638

\section{Summary of the protocol}

For the purpose of this trial, the interventions will be delivered in any hospital ward caring for COVID-19 patients at the participating centres. 


\begin{tabular}{|c|c|}
\hline Study Type : & Interventional (Clinical Trial) \\
\hline Sample size: & 364 participants \\
\hline Allocation: & Randomized \\
\hline Intervention Model: & Parallel Assignment \\
\hline $\begin{array}{l}\text { Intervention Model } \\
\text { Description: }\end{array}$ & Unblinded parallel-group randomized multicenter clinical trial \\
\hline Masking: & Open Label \\
\hline Primary Purpose: & Treatment \\
\hline Official Title: & $\begin{array}{l}\text { High-Flow Nasal Therapy Versus Conventional Oxygen Therapy in Patients With COVID-19: A Randomized } \\
\text { Controlled Trial (The COVID-HIGH Trial) }\end{array}$ \\
\hline $\begin{array}{l}\text { ClinicalTrials.gov } \\
\text { Identifier: }\end{array}$ & NCT04655638 \\
\hline
\end{tabular}

Table 1. Summary of the protocol

\section{Population}

Inclusion criteria to consider patients eligible will be:

- Age $\geq 18$ years old

- Tested positive for SARS-CoV-2 using real-time reverse transcriptase PCR (RT-PCR) nasopharyngeal swabs

- Clinical signs of acute respiratory infection and radiological evidence of pneumonia

- Hospital admission in any ward or Emergency Department within $48 \mathrm{~h}$

- $\mathrm{SpO}_{2} \leq 92 \%$ or $\mathrm{PaO}_{2} / \mathrm{FiO}_{2}<300$ in room air and need for oxygen therapy according to clinical judgment, at the screening.

Exclusion Criteria will be:

- $\mathrm{PaO}_{2} / \mathrm{FiO}_{2} \leq 200$

- Respiratory rate $\geq 28$ breaths/min and or severe dyspnea and or use of accessory muscles

- Need for immediate intubation or noninvasive ventilation (including CPAP) according to clinical judgment (e.g. clinical diagnosis of cardiogenic pulmonary edema, respiratory acidosis $\mathrm{pH} \leq 7.3$ )

- Patients already on CPAP/NIV or HFNT at study screening

- Septic shock

- Evidence of multiorgan failure

- Glasgow Coma Scale < 13

- Inability to comprehend the study content and give informed consent

- $\mathrm{PaCO}_{2}>45 \mathrm{mmHg}$, (if blood gas available) or history of chronic hypercapnia

- Patient already on long-term oxygen therapy (LTOT) or home NIV/CPAP (even if only overnight)

- Neuromuscular disease

- Limitation of care based on patients' or physicians' decision 


\section{Intervention and comparison}

The interventions under investigation will be high flow nasal therapy in comparison with conventional oxygen therapy.

HFNT will be delivered by any device (standalone machine or ventilators able to deliver it). The initial flow rate will be set at $40 \mathrm{~L} / \mathrm{min}$ and potentially increased up to $60 \mathrm{~L} / \mathrm{min}$, according to patient tolerance. Largebore nasal prongs will be selected according to the size of patients' nostrils (i.e. 2/3 of the diameter of the patient's nostril). A surgical mask will be placed on top of the HFNT interface. The temperature will be set at $37^{\circ} \mathrm{C}$ or $34{ }^{\circ} \mathrm{C}$ according to the patient's comfort. The FiO2 will be adjusted to maintain $\mathrm{SpO}_{2}$ between 92-96\%. A feeding tube or a nasogastric tube will not represent a contraindication for the use of HFNT provided the patency of the used nostril.

Conventional Oxygen therapy will be delivered by any device or combination of devices used for delivering oxygen such as nasal cannula, Venturi Mask or Mask with or without a reservoir bag as per usual local practice. Oxygen flow will be titrated to achieve $\mathrm{SpO}_{2}$ between $92-96 \%$.

Patients potentially eligible for the study will be evaluated by the attending physicians and receive medical therapy based on the attending physician's decision and local protocols. Awake proning is allowed. Local protocols, including drugs and awake proning, will be discussed with the enrolling centers at the initiation visit, and adherence to WHO guidelines will be recommended. Written informed consent from all the patients will be collected.

\section{Termination criteria \& protocol violation}

Criteria for weaning off COT or HFNT was at clinical discretion of the managing physician based on the improvement in oxygenation with ability to maintain $\mathrm{SpO}_{2}$ of $96 \%$ or greater with less than 0.30 of $\mathrm{FiO}_{2}$ or P/F > 300. The switch from COT to HFNT should be considered a protocol violation and should be based on clinical decision of the treating physician.

Criteria to be considered for escalation of treatment: 1) $\mathrm{SpO}_{2} \leq 92 \%$ despite COT or HFNT or P/F $\leq 180$ with $\mathrm{FiO}_{2} \geq 50 \%$, and 2) at least one of the following: respiratory rate $\geq 28$ breaths/min, severe dyspnea, signs of increased work of breathing (e.g. use of accessory muscles). If the patient meets these criteria, escalation of treatment CPAP, NIV or IMV will be considered.

The choice of the type of escalating treatment will be a clinical decision of the treating physician.

\section{Outcomes}

The primary outcome will be the proportion of patients needing escalation of treatment (i.e. noninvasive ventilation - including CPAP - or intubation) during hospital stay.

Secondary outcomes will be i) the proportion of patients needing intubation during hospital stay, ii) the proportion of patients who receive CPAP during hospital stay, iii) the proportion of patients who receive continuous positive airway pressure during hospital stay, iv) the proportion of patients who receive NIV during hospital stay, v) the proportion of patients undergone noninvasive ventilation (e.g. BiLevel, PSV), vi) the proportion of patients admitted to intensive care unit during hospital stay, vii) the proportion of 
patients who terminate the study protocols for improvement, viii) length of stay in hospital, ix) time to escalation of treatment to CPAP/NIV during hospital stay, $x$ ) time to escalation of treatment to intubation/invasive ventilation during hospital stay, xi) length of stay in ICU, xii) days free from CPAP/NIV during hospital stay, xiii) ventilator-free days during hospital stay, xiv) oxygen-free days during hospital stay, xv) 28-day mortality, xvi) 60-day mortality, xvii) hospital mortality, xix) treatment interruption due to intolerance during study treatment, $\mathrm{xx}$ ) dyspnea score (BORG scale) during hospital stay [0= no dyspnea to $10=$ severe dyspnea], xxi) National Early Warning Score 2 (NEWS2) during hospital stay, xxii) ROX index during hospital stay. 